\title{
iCELLigence real-time cell analysis system for examining the cytotoxicity of drugs to cancer cell lines (Review)
}

\author{
LEYLA TÜRKER ŞENER $^{1}$, GÜRCAN ALBENIZ ${ }^{2}$, BİRCAN DINÇ ${ }^{3}$ and IŞIL ALBENIZ ${ }^{1}$ \\ ${ }^{1}$ Department of Biophysics, Faculty of Medicine, Istanbul University, 34093 Istanbul; \\ ${ }^{2}$ Department of General Surgery, Cerrahpaşa Faculty of Medicine, Istanbul University, 34096 Istanbul; \\ ${ }^{3}$ Department of Basic Sciences, School of Engineering and Architecture, \\ Istanbul Kemerburgaz University, 34394 Istanbul, Turkey
}

Received September 26, 2016; Accepted March 23, 2017

DOI: $10.3892 / \mathrm{etm} .2017 .4781$

\begin{abstract}
The recently developed iCELLigence ${ }^{\mathrm{TM}}$ real-time cell analyzer (RTCA) can be used for the label-free real-time monitoring of cancer cell proliferation, viability, invasion and cytotoxicity. The RTCA system uses 16-well microtiter plates with a gold microelectrode biosensor array that measures impedance when cells adhere to the microelectrodes causing an alternating current. By measuring the electric field generated in this process, the RTCA system can be used for the analysis of cell proliferation, viability, morphology and migration. The present review aimed to summarize the working method of the RTCA system, in addition to discussing the research performed using the system for various applications, including cancer drug discovery via measuring cytotoxicity.
\end{abstract}

\section{Contents}

1. Introduction

2. RTCA system measurements

3. Cellular impedance and cell index

4. RTCA applications, advantages and steps

5. Using RTCA systems to measure the cytotoxicity of compounds to cancer cells

6. Conclusions

Correspondence to: Professor Işil Albeniz, Department of Biophysics, Faculty of Medicine, Istanbul University, 34093 Istanbul, Turkey

E-mail: ialbeniz@istanbul.edu.tr

Abbreviations: RTCA, real-time cell analyzing system; CI, cellular index

Key words: xCELLigence, iCELLigence, cytotoxicity, proliferation, cellular impedance, MCF-7, MDA-MB-231

\section{Introduction}

In 1984 Giaever and Keese (1) identified that cellular behavior could be investigated by applying an electric current to mammalian fibroblast cell culture via electrodes. Giaever and Keese applied an alternating electric field at $4,000 \mathrm{~Hz}$ to the fibroblast cell culture and measured a voltage drop between the boundary of the cell culture and the small electrode. The voltage drop at the boundary was affected by impedance and changed according to cell movement.

Real-time cell analyzing (RTCA) systems use gold microelectrode biosensors in each well of microtiter plates to measure electrical impedance. The weak electrical potential $(22 \mathrm{mV})$ and gold microarray surfaces are harmless to cell health and behavior. The electrical potential creates an electrical field between the cells and microelectrodes. Increasing the number of adherent cells and changing conditions in the cell culture alter the impedance. The impedance gives quantitative information about the number, viability, morphology and migration of the cells. RTCA does not require the labeling of cells, and overcomes the limitation of end-point data and incompatibility with orthogonal assays $(2,3)$. The RTCA iCELLigence $^{\mathrm{TM}}$ system (ACEA Biosciences, Inc., San Diego, CA, USA) detects biological events such as proliferation, invasion or apoptosis occurring in cells and provides dynamic impedance-based monitoring simultaneously $(4,5)$.

\section{RTCA system measurements}

xCELLigence $^{\mathrm{TM}}$ and iCELLigence products (both ACEA Biosciences, Inc.) use the RTCA system, which is a dynamic, real-time, label-free, cell-based microelectronic biosensor system that has applications in drug development, toxicology, microbiology, virology and cancer research (6,7).

xCELLigence and iCELLigence technology has been used to examine the behavior of cancer cells, including proliferation, migration and invasion in real-time at a cell culture level (8). At present, RTCA xCELLigence and iCELLigence products use the same technology. Well volumes in xCELLigence systems range from between 95 and $244 \mu \mathrm{l}$ in 16,36 and 96-well plates, and the resulting data can be stored and analyzed using desktop computers and notebooks (8). Well volumes in iCELLigence 


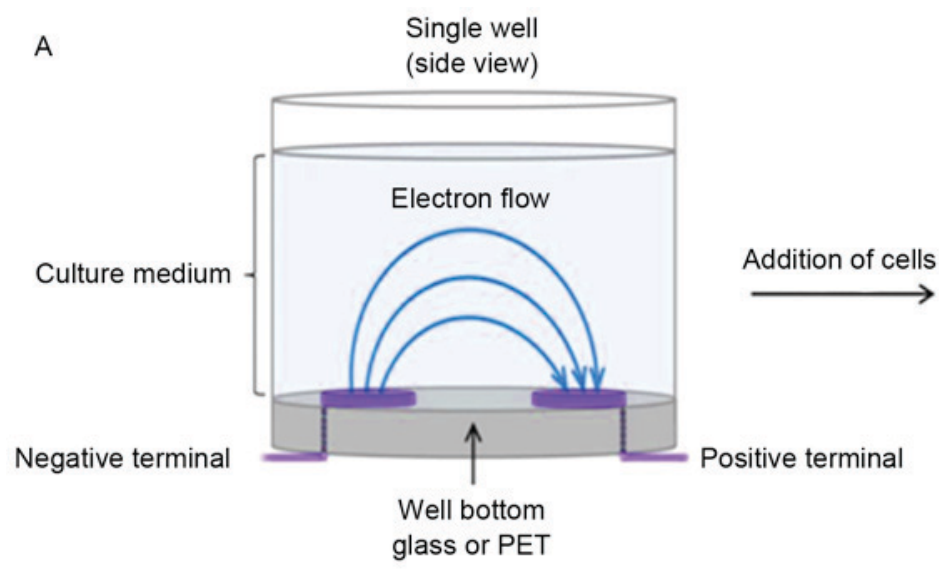

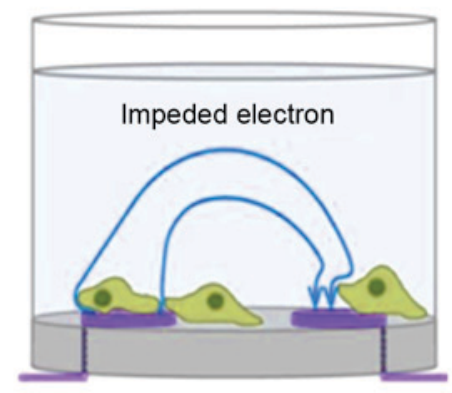

Figure 1. Impedance-based detection of cell viability and motility using a real-time cell analysis system. Illustration of the side view of a single well (A) prior to and (B) after cell addition.

systems are $830 \pm 10 \mu 1$ in 16 -well-plates and the resulting data is transferred to a tablet computer $(3,4)$. These RTCA systems measure alternate impedance caused by adherent cells in using interacting microelectrode sensor arrays placed at the bottom of electronic (E)-plate inserts (9). The system obtains real-time results that reflect the adhesion, proliferation, apoptosis, propagation and morphologic changes of cells over long periods of time (10).

\section{Cellular impedance and cell index}

The impedance of gold microelectrodes in RTCA systems when cells are not present or not adhered onto the electrodes is determined with ionic cell culture medium solution. Adherent cells act as an insulator on the surface of the electrode and change the ionic medium of the electrode solution, increasing the impedance (11). The higher the proliferation and adhesion rate of the cells, the more increased the impedance is (Fig. 1). The cell index (CI) is a function of the cell number and ratio of cells at different time intervals; $\mathrm{CI}=0$ when there is no cell adhesion. The CI in a RTCA system is the result of the impedance induced by adherent cells to the electron flow. $\mathrm{CI}$ is calculated as follows: $\mathrm{CI}=$ (impedance at time point $\mathrm{n}$-impedance in the absence of cells)/nominal impedance value.

Prior to the addition of cells, electric current flows freely through the culture medium in a RTCA system. Following cell adhesion and proliferation, current flow is impeded, which provides a sensitive readout of cell number, size and morphology, viability, migration and substrate attachment quality $(2,6,12)$.

\section{RTCA applications, advantages and steps}

In the first step of an RTCA assay, cancer cells are seeded into the wells of a microtiter E-plate. Due to proliferation causing an increase in adherent cells, the electric current becomes increasingly impeded. This impedance value is plotted as the CI value (13). In the second step the effector matter being investigated is added to the wells. For examples, plant extract can be used as the effector matter (13). This added matter changes the CI and decreases the proliferation ratio (Fig. 2). The RTCA system is convenient for investigating interactions between plant extracts and cancer cells (14). This method decreases contamination risk, and enables extra functionality compared with other classic colometric tests such as MTT, MTS, XTT and WST (Fig. 3). The RTCA system has applications in numerous areas, including stem cell, cancer and drug research.

Prior to the addition of cells, a wireless connection between the iCELLigence system and tablet computer is opened. Software of the xCeLLigence RTCA (ACEA Biosciences, Inc.) is opened and used according to the manufacturer's protocol for the appropriate application; for example, measuring cytotoxicity. The user can change the duration of intervals between measurements, and input the number of cells seeded and effector concentration for each well. After these adjustments, $150 \mu \mathrm{l}$ of the appropriate cell medium is added to the wells and a background value is taken. The cells are then added; if some wells do not reach necessary CI value of 1 , more cells can be added. After this stage, the effector matter is added. After these steps, the experiments continue according to the adjusted parameters and data is collected. As a result of the assay, CI values are obtained, in addition to half maximal inhibitory concentration values for cytotoxicity applications (15).

\section{Using RTCA systems to measure the cytotoxicity of compounds to cancer cells}

Research has been conducted to examine the behavior of cancer cells, including proliferation, adhesion, invasion and migration, in real-time on a cell culture level (4-6). Milk fat globule epidermal growth factor VIII (MFG-E8) protein increases the survival of breast cancer, melanoma and bladder carcinoma subtype cells (16). The effect of anti-MFG-E8 antibodies on the proliferation, migration and survival of cancer cells can be evaluated using a RTCA system (16). These antibodies can then be recommended as novel cancer treatments.

The effect of 6-bromo-indirubin (BIO) was investigated using an RTCA system. BIO inhibits the adhesion, proliferation and metastasis of T24, HuH-7, MDA-MB-231 and murine mammary carcinoma cell lines (17). RTCA has also been used to explore the metastasis of ovarian cancer cells 
A

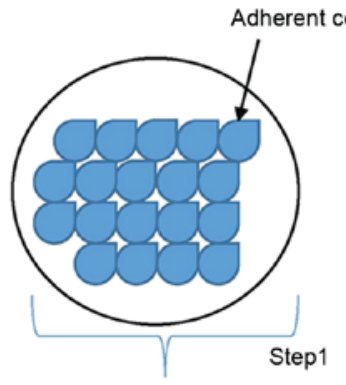

Gold microelectrodes

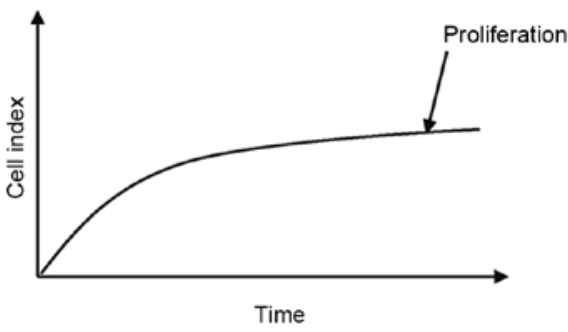

B
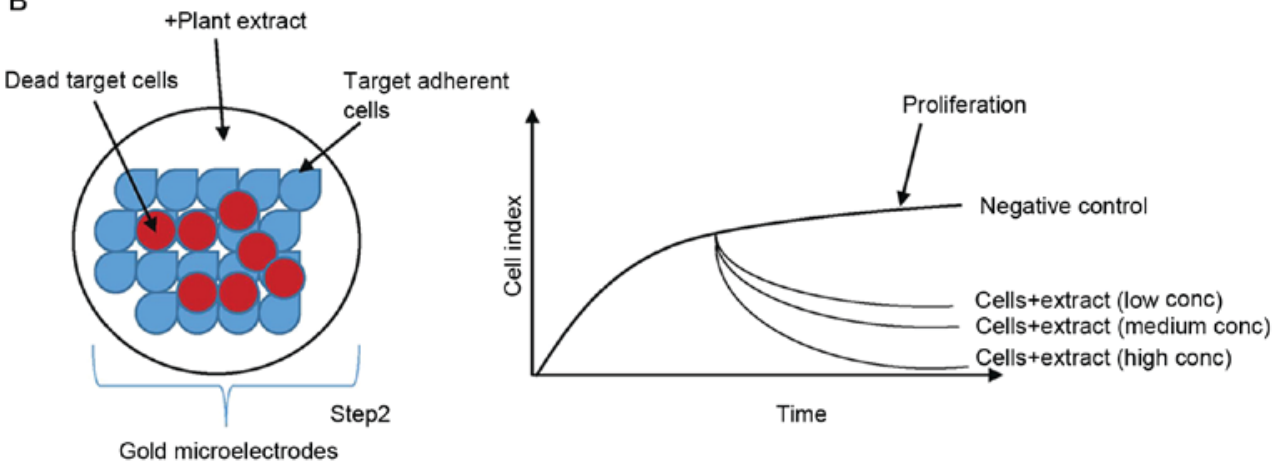

Figure 2. Cytotoxicity assay using a real-time cell analysis system. (A) Prior to and (B) after the addition of an effector; for example, plant extract.

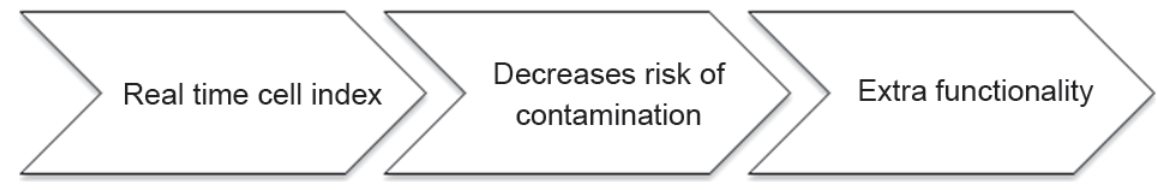

Figure 3. Advantages of real-time cell analysis.

lines grown as spheroids in peritoneal fluid and distal sites within the peritoneum (18). In the study, measurements were taken continuously over a long period using a RTCA system to examine interactions between the extracellular matrix barrier and the cell layer, which overcomes the requirement for exhaustive repetitive microscopic examinations (18). Breast cancer is the second leading cause of cancer-associated mortality in women and 1 million new cases of breast cancer are diagnosed annually, as reported in 2017 (19). RTCA is being used to identify novel drugs for the treatment of breast cancer. A platinum II complex was identified to inhibit MCF-7 and MDA-MB-231 breast cancer cell growth, with MDA-MB-231 cells being more sensitive to the complex compared with MCF-7 cells (20). Another study using RTCA investigated the long-term effect of different doses of aluminum on MDA-MB-231 cells (21). The results of this study indicated that aluminum contributes to the development of breast cancer. In another long-term study (23 weeks) using RTCA, aluminum considerably increased the proliferation of MDA-MB-231 cells (22). RTCA has also been used to examine the effect of Vitamin $\mathrm{K}$ derivatives on the adhesion and proliferation of MDA-MB-231 and human epidermal growth factor 2-positive breast cancer cell lines $(23,24)$. Vitamin $\mathrm{K}$ derivatives were revealed to decrease the adhesion and proliferation of the cells, highlighting the importance of nutrition in the treatment of cancer.

Research into the effect of natural agents in cancer is increasing and numerous anticancer drugs originate from plants. Thus, investigating the anticancer effects of medicinal plant extracts may provide compounds that can be used for the treatment of cancer. Flavones, including apigenin and luteolin, that are present in crop and herbs have antiallergic, anti-inflammatory, antioxidant and antitumor effects (25). The effect of flavones on MCF-7 breast cancer cells was evaluated by performing cell migration analysis using a RTCA system (25). Different concentrations of flavones were applied to the cells and measurements were taken every hour for $9 \mathrm{~h}$, revealing that flavones significantly increased cancer cell apoptosis compared with the control group. This suggests that flavone compounds have a potential application in the prevention and treatment of cancer. Studies have investigated the cytotoxicity and antiproliferative activities of extracts obtained from various herbs $(26,27)$. The in vitro cytotoxic effects of numerous herbal extracts have been investigated on MDA-MB-231 and MCF-7 breast cancer cells (28). The cytotoxic effect of plant extracts (Thymus serpyllum) on MDA-MB-231 cells was measured using an RTCA iCELLigence system (Fig. 4). An example of a real-time impedance 


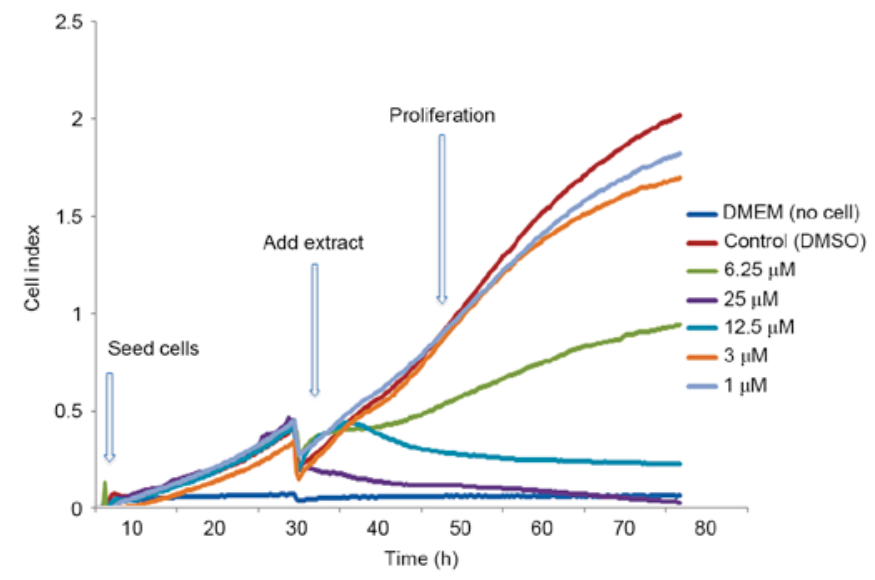

Figure 4. Real-time cell analysis of increasing concentrations of plant extract on MDA-MB-231 cells.

trace throughout a cytotoxicity experiment is presented in Fig. 2.

\section{Conclusions}

Cytotoxicity is defined as the property of a compound to kill cells. Cytotoxicity assays can be performed to determine the toxic effect of active substances on cancer cells. Cytotoxic agents remain the preferred treatment for numerous types of cancer (29). Cytotoxic chemotherapy agents affect cells that are in mitosis more compared with those in G0 (30). Cytotoxicity assays to assess the potential of anticancer compounds should search for the lowest cytotoxic concentration. Cytotoxic drugs interfere with the function of intracellular signaling molecules, membrane receptors and kinases that are responsible for the proliferation of cells, inhibiting cell growth and division prior to inducing cell death (31).

For the determination of cytotoxicity, various analysis methods are used. Conventionally, trypan blue, lactate dehydrogenase, neutral red uptake, MTT, MTS, WST-1 and XTT assays can be used for the determination of cell viability and cytotoxicity. In addition, RTCA systems can now be used to measure cytotoxicity. In comparison with image acquisition using microscopes, RTCA allows the analysis of the whole period of the experiment and does not require the labeling that can negatively affect cell culture experiments. The RTCA iCELLigence system allows researchers to choose data collection intervals and record real-time data. This is particularly important for long-term experiments, including those investigating aluminum compounds that can continue for weeks. RTCA systems provide an easy method of analyzing cell cultures. The RTCA iCELLigence system is a form of the RTCA xCELLigence system; they use the same technology but iCELLigence gives users the opportunity to work with larger well volumes and fewer wells.

The RTCA iCELLigence system is a microelectronic biosensor system for cell-based assays, providing dynamic, real-time, label-free cellular analysis for a variety of research applications, including drug development, toxicology, cancer, medical microbiology and virology. RTCA has taken the place of conventional assays for cellular activities, including the MTT and trypan blue assays, with high sensitivity and specificity. In conclusion, the RTCA iCELLigence system can be used to investigate the interactions between compounds and cells, aiding in the development of novel drugs. The RTCA iCELLigence is likely to become increasingly used for a wide range of applications in the near future.

\section{References}

1. Giaever I and Keese CR: Monitoring fibroblast behavior in tissue culture with applied electric field. Proc Natl Acad Sci USA 81: 3761-3764, 1984.

2. Cooper MA: Non-optical screening platforms: The next wave in label-free screening? Drug Discov Today 11: 1068-1074, 2006.

3. Garcia SN, Gutierrez L and McNulty A: Real-time cellular analysis as a novel approach for in vitro cytotoxicity testing of medical device extracts. J Biomed Mater Res A 101: 2097-2106, 2013.

4. Ke N, Wang X, Xu X and Abassi YA: The xCELLigence system for real-time and label-free monitoring of cell viability. Methods Mol Biol 740: 33-43, 2011.

5. Giaever I and Keese CR: A morphological biosensor for mammalian cells. Nature 366: 591-592, 1993.

6. Oktem O, Bildik G, Senbabaoglu F, Lack NA, Akin N, Yakar F, Urman D, Guzel Y, Balaban B, Iwase A and Urman B: Cytotoxicity and mitogenicity assays with real-time and label-free monitoring of human granulosa cells with an impedance-based signal processing technology intergrating micro electronics and cell biology. Reprod Toxicol 60: 82-91, 2016.

7. Teng Z, Kuang X, Wang J and Zhang X: Real-time cell analysis-a new method for dynamic, quantitative measurement of infectious viruses and antiserum neutralizing activity. J Virol Methods 193: 364-370, 2013.

8. Limame R, Wouters A, Pauwels B, Fransen E, Peeters M Lardon F, De Wever O and Pauwels P: Comparative analysis of dynamic cell viability, migration and invasion assessments by novel real-time technology and classic endpoint assays. PLoS One 7: e46536, 2012.

9. Benachour H, Bastogne T, Toussaint M, Chemli Y, Sève A, Frochot C, Lux F, Tillement O, Vanderesse R and Barberi-Heyob M: Real-time monitoring of photocytotoxicity in nanoparticles-based photodynamic therapy: A model-based approach. PLoS One 7 e48617, 2012.

10. Kustermann S, Boess F, Buness A, Schmitz M, Watzele M, Weiser T, Singer T, Suter L and Roth A: A label-free, impedance-based real time assay to identify drug-induced toxicities and differentiate cytostatic from cytotoxic effects. Toxicol In Vitro 27: 1589-1595, 2013.

11. Szulcek R, Bogaard HJ and van Nieuw Amerongen GP: Electric cell-substrate impedance sensing for the quntification of endothelial proliferation, barrier function, and motility. J Vis Exp: Mar 28, 2014. doi: 10.3791/51300.

12. Kirstein SL, Atienza JM, Xi B, Zhu J, Yu N, Wang X, Xu X and Abassi YA: Live cell quality control and utility of real-time cell electronic sensing for assay development. Assay Drug Dev Technol 4: 545-553, 2006.

13. Ozdemir A and Ark M: xCELLigence real time cell analysis system: A new method for cell proliferation and cytotoxicity. Niche 2: 15-17, 2013.

14. Catalano E, Maestri P, Cochis A, Azzimonti B, Varoni EM, Fraschini F, Rimondini L, Megna S and Iriti M: Cytotoxic activity of a plant extract on cancer cells. Italian J Anatomy Embriyol 118: 50, 2013.

15. Moodly K, Angel CE, Glass M and Graham ES: Real time profiling of $\mathrm{NK}$ cell killing of human astrocytes using xCELLigence technology. J Neurosci Methods 200: 173-180, 2011.

16. Tibaldi L, Leyman S, Nicolas A, Notebaert S, Dewulf M, Ngo TH, Zuany-Amorim C, Amzallag N, Bernard-Pierrot I, Sastre-Garau X and Théry C: New blocking antibodies impede adhesion, migration and survival of ovarian cancer cells, highlighting MFGE8 as a potential therapeutic target of human ovarian carcinoma. PLos One 8: e72708, 2013

17. Braig S, Kressirer CA, Liebl J, Bischoff F, Zahler S, Meijer L and Vollmar AM: Indirubin derivative 6BIO suppresses metastasis. Cancer Res 73: 6004-6012, 2013.

18. Bilandzic M and Stenvers KL: Assessment of ovarian cancer spheroid attachment and invasion of mesothelial cells in real time. J Vis Exp: May 20, 2014 doi: 10.3791/51655.

19. Breastcancer.org: U.S. Breast Cancer Statistics. http://www. breastcancer.org/symptoms/understand_bc/statistics. Accessed March 10, 2017. 
20. Oral AY, Cevatemre B, Sarimahmut M, Icsel C, Yilmaz VT and Ulukaya E: Anti-growth effect of a novel trans-dichloridobis [2-(2-hydroxyethyl)pyridine]platinum (II) complex via induction of apoptosis on breast cancer cell lines. Bioorg Med Chem 23: 4303-4310, 2015.

21. Darbre PD: Aluminium, antiperspirants and breast cancer. J Inorg Biochem 99: 1912-1919, 2005.

22. Bakir A and Darbre PD: Effect of aluminium on migration of oestrogen unresponsive MDA-MB-231 human breast cancer cells in culture. J Inorg Biochem 152: 180-185, 2015.

23. Kiely M, Hodgins SJ, Merrigan BA, Tormey S, Kiely PA and O'Connor EM: Real-time cell analysis of the inhibitory effect of vitamin $\mathrm{K} 2$ on adhesion and proliferation of breast cancer cells. Nutr Res 35: 736-743, 2015.

24. Hashemi HB, Karimi S and Mahboobi H: Lifestyle changes for prevention of breast cancer. Electron Physician 6: 894-905, 2014.

25. Lin CH, Chang CY, Lee KR, Lin HJ, Chen TH and Wan L: Flavones inhibit breast cancer proliferation through the Akt/FOXO3a signaling pathway. BMC Cancer 15: 958, 2015.

26. Yagloglu AS, Eser F, Tekin S and Onal A: Antiproliferative activities of several plant extracts from Turkey on rat brain tumor and human cervix carcinoma cell lines. Front Sci 9: 69-74, 2016.
27. Nunes R, Pasko P, Tyszka-Czochara M, Szewczyk A, Szlosarczyk M and Carvalho IS: Antibacterial, antioxidant and anti-proliferative properties and zinc content of five south Portugal herbs. Pharm Biol 55: 114-123, 2017.

28. Abdullah AS, Mohammed AS, Abdullah R, Mirghani ME and Al-Qubaisi M: Cytotoxic effects of Mangifera indica L. kernel extract on human breast cancer (MCF-7 and MDA-MB-231 cell lines) and bioactive constituents in the crude extract. BMC Complement Altern Med 14: 199, 2014.

29. Martinez-Serra J, Gutierrez A, Muñoz-Capó S, Navarro-Palou M, Ros T, Amat JC, Lopez B, Marcus TF, Fueyo L, Suquia AG, et al: xCELLigence system for real-time label-free monitoring of growth and viability of cell lines from hematological malignancies. Onco Targets Ther 7: 985-994, 2014.

30. Mycek MJ, Harvey RA, Champe PC and Cooper BD: Lippincott illustrated reviews. In: Pharmacology. Lippincott, Williams \& Wilkins, Philadelphia, PA, 2000.

31. Elmore S: Apoptosis: A review of programmed cell death. Toxicol Pathol 35: 495-516, 2007. 